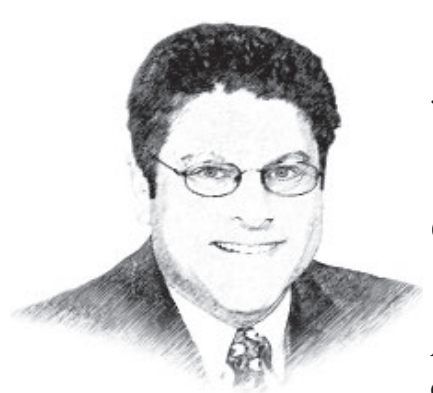

\title{
Heightening awareness of blistering disorders
}

Among the different patterns of skin disease, blistering disorders link to one of the more diverse and potentially confusing sets of etiologies that confront nondermatologists. The spectrum of associated disorders ranges from annoying to life-threatening, and prompt suspicion and biopsy with direct immunofluorescence may be necessary for appropriate diagnosis and treatment.

The distribution of vesicular or bullous lesions, the clinical setting, medications, environmental exposures, and coexistent symptoms all come into play as the initial differential diagnosis is generated. Regional blistering lesions can occur with various triggers of contact dermatitis from adhesive tape and iodine, to plant-triggered "poison oak," and even bug bites under certain circumstances. (Consider underlying hematologic malignancy or HIV with particularly prolonged or disseminated reactions.)

We are all familiar with the localized, often recurrent vesicles of herpes simplex, as well as the crops of vesicular lesions with herpes zoster. Both can disseminate in patients lacking immunocompetence. The coincident odd occurrence of classic erythema multiforme on palms or soles with localized vesicular herpes infection elsewhere can sometimes cause initial diagnostic confusion, as all lip blisters are not herpetic.

Another viral infection causing vesicular lesions with "hand, foot, and mouth disease," particularly in children, is from a coxsackievirus that can also affect adults, causing some confusing systemic symptoms. Localized pruritic papulovesicular patches are the cardinal lesions in dermatitis herpetiformis, and they may occur without any other clinical features of the associated gluten sensitivity. And then there are the severe, lifethreatening, diffuse, blistering, peeling allergic reactions to medications characterized as toxic epidermal necrolysis and Stevens-Johnson syndrome.

I highlight these few examples not as a comprehensive review, but because they have been the more common considerations I have had when seeing patients with vesicles and blisters. On reading the paper by Mendoza et $\mathrm{al}^{1}$ in this issue of the Journal (page 319), I was reminded of some significant diagnoses that I may have not recognized as early as I might have.

Bullous pemphigoid is an autoimmune disease characteristically occurring in older individuals, and I think of it in that setting when I recognize the characteristic tense bullae. Its pathophysiology is understood as the generation of pathogenic autoantibodies against several defined skin basement membrane proteins, detected by routine immunofluorescence testing on biopsy of the blister and adjacent skin. The tense blisters-unlike the more superficial, often weeping, easily torn, and nonhealing lesions of (the more serious) pemphigus - are often surrounded by erythema. Many patients with bullous pemphigoid will describe a prodrome of significant patchy pruritus, sometimes with urticarial or eczematoid plaques, that may persist for weeks or even months prior to the appearance of the blisters and awareness of the likely diagnosis. Thus, pemphigoid should be included in the differential diagnosis for new-onset unexplained localized pruritus. 
There are also subsets of pemphigoid localized to mucous membranes that warrant aggressive therapy to prevent scarring. They include involvement of the conjunctivae (cicatricial pemphigoid), which if untreated can lead to visual impairment or blindness, and involvement of the nasal and upper airways that can mimic limited granulomatosis with polyangitis in its degree of local tissue damage. And there are several other forms characterized by specific locations and features.

While these autoimmune blistering diseases are uncommon, even in most dermatology practices, their potential severity and association with multiple systemic and often clinically disconnected features warrant our awareness.

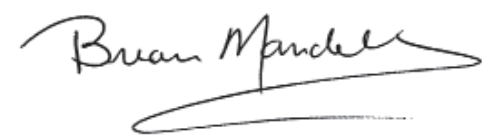

Brian F. Mandell, MD, PhD

Editor in Chief

1. Mendoza H, Goodwin J, Gehlhausen J, Odell I, McNiff J, Gnanapandithan K. New bullous lesions in a 72-year-old woman. Cleve Clin J Med 2021; 88(6):319-324. doi:10.3949/ccjm.88a.20180 